\title{
Study on Effect of Storage Time on Oxidation Induction Time of HDPE
}

\author{
Yun Cheng ${ }^{1, \text { a }}$, Xiaoli Zhao ${ }^{1, b}$, Weijun Zhong ${ }^{1, ~ c}$, Yanfeng Yang ${ }^{1, d}$ \\ ${ }^{1}$ Ordnance Engineering College, Shijiazhuang050003, China

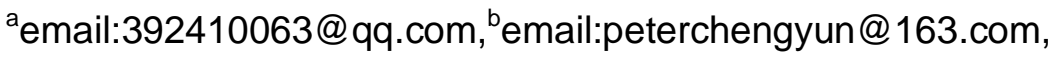 \\ cemail:zhwj81@sohu.com, ${ }^{\mathrm{c} e m a i l: y a n g y a n f e n g @ 126 . c o m ~}$
}

Keywords: High density polyethylene; Oxidation induction time; DSC; Storage time

Abstract. The performance of high density polyethylene products during storage will decline because of the aging of materials. The oxidation induction time is an important performance to reflect the oxidation resistance characterize of the material. The oxidation induction time of the samples during different periods is tested by the differential scanning calorimetry (DSC). The variation of the oxidation induction time with storage time is studied and analyzed. It is the basis of the further study on oxidation resistance of the high density polyethylene products.

\section{Introduction}

Polymer material, as a new material, has played a huge role in various industrial fields after half a century of development. However, the performance of polymer products will decline because of the aging during storage and use. What's more, polymer products will lose the value. Polymer products often play an important role in the application, especially in the national defense and military products. Once the aging failure occurs in service, it will cause not only economic losses, even may lead to personal injury or death, environmental damage and other accidents. Therefore we must research the aging behavior of products, and replace the parts which will be about to lose efficacy. So, it has the importance significance that evaluation of the anti-aging effects of antioxidants in the development and characterization of polymer materials ${ }^{[1]}$.

One grenade is one of important artilleries in close combat, mainly used for killing the enemy's effective strength and destroying the armored targets in close combat. It has the advantages of small size, light weight, simple structure, easy to carry, etc ${ }^{[2]}$. A large number of plastic materials are applied to the grenade. The fuze stock is made by HDPE, and the projectile body is made by LLDPE. After the long-time storage, varying degrees of aging will happen in the plastic material of the grenade, which will seriously affect the normal storage and use of the grenade.

In this paper, the oxidation induction time of the fuze stocks at different times are measured by differential scanning calorimetry, which provides some basis for the study on the antioxidant properties and quality transition of the material.

\section{Introduction of the oxidation induction time}

Oxidation induction time is an index to represent the oxidation resistance characterize of the material. Oxidation induction time (OIT) is the time that the measurement sample begins to autocatalytic oxidation at high temperatures $\left(200^{\circ} \mathrm{C}\right)$ oxygen conditions, which can evaluate the thermal degradation of the material in molding processing, storage, welding and the use. In recent years, development of modern testing technology and testing equipment, determining oxidation induction time of material utilizing differential scanning calorimetry (DSC) has become an 
important method to evaluate the thermal oxidative stability ${ }^{[3 \sim 5]}$. The method has the advantages for less sample, short test period and good reproducibility, and has a good relationship with other oxidation test. So the method is widely used in practical work ${ }^{[6 \sim 7]}$. Oxidation induction time is a method to test the degree of accelerated aging in high temperature oxygen, using the differential thermal analysis (DTA) based on the exothermic reaction when the molecular chains of plastic are ruptured. The principle is that the plastic sample and an inert reference material (such as aluminum oxide) are placed in a differential thermal analyzer, and the inert gas in sample chamber (such as nitrogen) is rapidly replaced by oxygen at a certain temperature. The changes of DTA curve (differential thermal spectrum) are tested due to sample oxidation, and the oxidation induction time (OIT) of the sample is obtained to assess the plastic antioxidant properties ${ }^{[8]}$.

\section{Experimental section}

Experimental material:

High density polyethylene; nitrogen; oxygen

Experimental apparatus:

Differential Scanning Calorimetry; open crucible.

Experimental procedure

Weigh the sample at a certain time, and put the sample into the open crucible. Ensure the full contact between the sample and the crucible bottom. Raise the test temperature to $200^{\circ} \mathrm{C}$ at the heating rate of $20 \mathrm{~K} / \mathrm{min}$ in a stream of nitrogen. After reaching the test temperature, stop the heating program and place the sample at the temperature for $3 \mathrm{~min}$.

After the constant time, immediately switch to the oxygen at the same flow rate with the nitrogen. The oxygen switching point is referred to as the zero test. Continue thermostat until the exothermic significant change point appears after $2 \mathrm{~min}$.

When the test is finished, switch the gas converter back to nitrogen and cool the instrument to room temperature. ${ }^{[9]}$

Take the samples during different times and repeat the above steps. Obtain the oxidation induction time of samples during different storage times.

\section{Experimental result}

The oxidation induction time of samples during different storage times

Take samples during different storage times in the North and the South to test the oxidation induction time. The test data in the North are shown in table 1, and the test data in the South are shown in table 2:

Table 1 The OIT of sample in the North

\begin{tabular}{ccccccccc}
\hline storage time[a] & 1 & 15 & 16 & 17 & 19 & 22 & 26 & 26 \\
\hline OIT[min] & 82.6 & 84.4 & 82.9 & 78.2 & 83.8 & 50.2 & 57.9 & 46.1 \\
\hline
\end{tabular}

Table 2 The OIT of sample in the South

\begin{tabular}{ccccccccccccc}
\hline storage time[a] & 1 & 12 & 17 & 19 & 19 & 22 & 24 & 24 & 26 & 26 & 29 & 29 \\
\hline OIT[min] & 82.6 & 85.9 & 54.3 & 83.5 & 85.9 & 64.2 & 56.1 & 53.2 & 39.7 & 53 & 52.7 & 40.5 \\
\hline
\end{tabular}




\section{Discussion}

The variation of the oxidation induction time with storage time

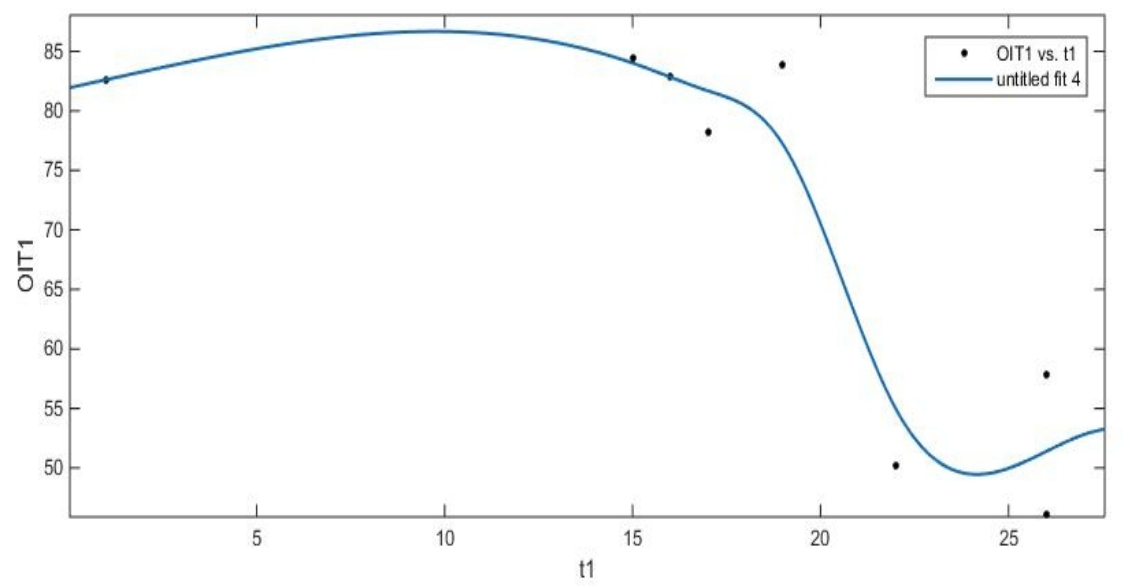

Fig. 1 Trend of OIT in the North

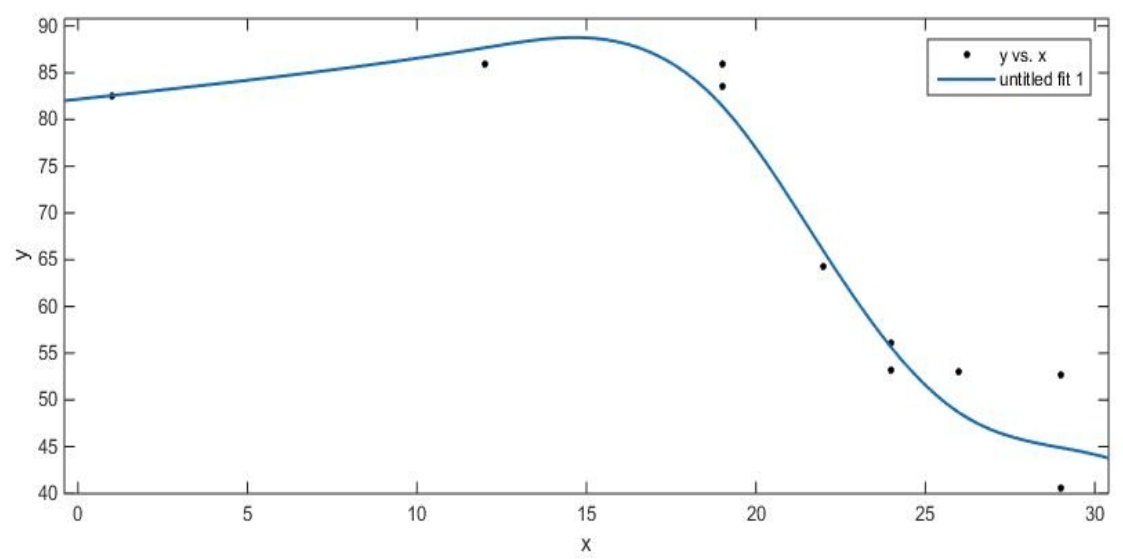

Fig. 2 Trend of OIT in the South

The trend of OIT in the North is shown in Fig.1. The trend of OIT in the South is shown in Fig.2. From the above figures, the oxidation induction time of the plastic samples in the North and South will be significantly reduced when the storage time increases, which indicates that the antioxidant properties of plastics samples have a significant decline. And the samples in the South and North have the same trend of performance degradation. From the data, the oxidation induction time don't change substantially when the plastic material storage within 19 years, which indicates that the property of the samples is excellent. The oxidation induction time and the oxidation resistance will reduce significantly, when the storage time of samples is greater than 22 years. It indicates that the performance of the plastic material will decrease during 19 to 22 years.

\section{Conclusions}

(1) Oxidation induction time of the plastic material will reduce significantly with storage time, which indicates that the antioxidant properties reduce significantly.

(2) The samples in the North and South have the same trend of performance degradation.

(3) The antioxidant properties of plastic materials reduce during the 19 to 22 years. 


\section{References}

[1] Qiukai Zheng, Peng Liu, Dehong Cheng. New Method of Evaluating Anti-Oxidation Property of Polypropylene[J]. Petrochemical Technology, 2009, 38(5):563 566.

[2] Shanwu Wang. Grenade Development Tendency and Prospects . Technology Foundation of National Defence,2008(10):45-50.

[3] Baojun Dong, Lijun Zhang, Hai Gao. Determination of oxidation induction time of polyolefin by DSC[J]. Plastics Technology and Equipment, 2010, 36(11): 25 27.

[4] Zhenghuan Li. Chinese and overseas test method standards of determination for oxidation induction time[J]. China Standardization, 2005, (4): 29 32.

[5] Phease T.L., Billingham N.C., Bigger S.W.. The effect of carbon black on the oxidative induction time of medium-density polyethylene[J]. Polymer, 2000, 41(26): 9123 9130.

[6] Lijun Zhang, Lijun Liu, Jinxian Jiang. Determination of plastic oxidation induction time and oxidation induction temperature by DSC[J]. Plastics Technology and Equipment, 2010, 36(6): 12 15.

[7] Lijun Zhang, Liwei Liang, Baojun Dong. Determination of ABS resin oxidation induction time by DSC[J]. Analytical Instrumentation, 2011, (1): 36 38.

[8] GB/T 19466.6-2009, Plastics-Differential scanning calorimetry(DSC)-Part 6: Determination of oxidation induction time(isothermal OIT) and oxidation induction temperature(dynamic OIT)[S].

[9] Wanjuan Wang, Guoshan He, Xiaoqiang Xiao. Effect of test conditions on oxidation induction time of polyethylene-based wood plastic composite material[J]. Plastics Technology and Equipment, 2014, 40(12):39 43. 